D. Hada, L. Sario, and C. Wang

Nagoya Math. J.

Vol. 54 (1974), 1-6

\title{
N-MANIFOLDS CARRYING BOUNDED BUT NO DIRICHLET FINITE HARMONIC FUNCTIONS
}

\author{
DENNIS HADA, LEO SARIO, AND CECILIA WANG
}

Among the most remarkable results in the theory of harmonic functions on Riemann surfaces is the strictness of the inclusion relations $O_{G}<O_{H B}<O_{H D}$, established by Ahlfors [1,2], Royden [2,4], and Tôki [8] two decades ago. Subsequently the strictness of the relations $O_{G}<$ $O_{H P}<O_{H B}$ was shown and a somewhat simpler proof of $O_{H B}<O_{H D}$ given by Sario [5] and Tôki [9]. Here $O_{G}$ is the class of parabolic surfaces, and $O_{H P}, O_{H B}, O_{H D}$ stand for the classes of surfaces which do not carry nonconstant harmonic functions which are positive, bounded, or Dirichlet finite, respectively. The corresponding nonstrict inclusion relations extend readily to Riemannian manifolds of any dimension, and so does the strictness of $O_{G}<O_{H P}<O_{I I B}$ (see e.g. Sario-Schiffer-Glasner [7] and SarioNakai [6]). In contrast, the strictness of $O_{H B}<O_{H D}$ has remained an open problem. The purpose of the present paper is to submit an example which solves the problem in the affirmative for an arbitrary dimension $N$. In the process we also obtain complete characterizations of the Poincaré $N$-ball in $O_{H B}$ and $O_{H D}$. This manifold plays an important role in the harmonic and biharmonic classification theory.

1. For $N \geq 3$, consider the $N$-ball $B_{\alpha}^{N}=\left\{|x|<1, x=\left(x^{1}, \cdots, x^{N}\right), d s\right\}$ with the Poincaré-type metric $d s=\left(1-|x|^{2}\right)^{\alpha}|d x|, \alpha$ constant.

THEOREM 1. $B_{\alpha}^{N} \notin O_{H B} \Leftrightarrow \alpha<1 /(N-2), N \geq 3$.

Proof. For the necessity, observe that a radial function $h(r), r=$ $|x|$, is harmonic if and only if

Received November 16, 1972.

The work was sponsored by the U.S. Army Research Office-Durham, Grant DA-AROD-31-124-71-G181, University of California, Los Angeles.

MOS classification 31B05. 


$$
\Delta h(r)=-\left(1-r^{2}\right)^{-N \alpha} r^{1-N} \frac{d}{d r}\left[\left(1-r^{2}\right)^{(N-2) \alpha} r^{N-1} h^{\prime}(r)\right]=0,
$$

that is,

$$
h(r)=\mathrm{const} \int_{a}^{r}\left(1-r^{2}\right)^{-(N-2) \alpha} r^{1-N} d r .
$$

Since $B_{\alpha}^{N} \in O_{G}$ if and only if $|h(r)| \rightarrow \infty$ as $r \rightarrow 1$, the parabolicity is characterized by $\alpha \geq 1 /(N-2)$. In view of $O_{G} \subset O_{H B}$, we have the necessity. To prove the sufficiency, let $\left\{S_{n m}\right\}$ be the spherical harmonics. First we find an $f_{n}(r)$ such that $f_{n} S_{n m}$ is harmonic on $B_{\alpha}^{N}$. The equation $\Delta\left(f_{n} S_{n m}\right)=0$ gives

$$
\begin{gathered}
\left(1-r^{2}\right) r^{2} f_{n}^{\prime \prime}(r)+\left\{N-1-[(N-1)+2(N-2) \alpha] r^{2}\right\} r f_{n}^{\prime}(r) \\
-n(n+N-2)\left(1-r^{2}\right) f_{n}(r)=0 .
\end{gathered}
$$

The origin is a regular singular point, and there exists a solution of the form $f_{n}(r)=\sum_{i=0}^{\infty} c_{n, i} r^{n+i}$ with $c_{n, 0}=1$. A recursion formula for the coefficients is obtained in the usual manner and yields (cf. [3])

$$
c_{n, 2 i}=\prod_{j=1}^{i} \frac{(n+2 j-2)[n+2 j+N-4+2(N-2) \alpha]-n(n+N-2)}{(n+2 j)(n+2 j+N-2)-n(n+N-2)},
$$

$c_{n, 2 i+1}=0$ for $i \geq 0$. We shall show that $f_{n} S_{n m} \in H B\left(B_{\alpha}^{N}\right)$ if $\alpha<1 /(N-2)$.

There exists an $i_{0}=i_{0}(N, \alpha)$ such that for $j \geq i_{0}$ and $\alpha<1 /(N-2)$, $c_{n, 2 j} / c_{n, 2 j-2}$ is positive and dominated by

$$
\frac{(n+2 j-2)[n+2 j+N-4+2(N-2) \alpha]}{(n+2 j)(n+2 j+N-2)} .
$$

For $i \geq i_{0}$,

$$
\begin{aligned}
\left|c_{n, 2 i}\right| & \left.<c \prod_{j=1}^{i} \frac{n+2 j-2}{n+2 j} \cdot \prod_{j=i_{0}}^{i} \frac{n+2 j+N-4+2(N-2) \alpha}{n+2 j+N-2}\right) \\
& =\frac{c n}{n+2 i} \prod_{j=i_{0}}^{i}\left(\frac{2-2(N-2) \alpha}{n+2 j+N-2}\right) .
\end{aligned}
$$

Here and later, $c$ is a positive constant, not always the same. We now impose on $i_{0}$ the additional condition that for $j \geq i_{0}$

$$
0<\frac{2-2(N-2) \alpha}{n+2 j+N-2}<1
$$

Then 


$$
\begin{aligned}
\sum_{j=i_{0}}^{i} \log \left(1-\frac{2-2(N-2) \alpha}{n+2 j+N-2}\right) & <-2[1-(N-2) \alpha] \\
\times \sum_{j=i_{0}}^{i} \frac{1}{n+2 j+N-2} & <-[1-(N-2) \alpha] \int_{i_{0}}^{i+1} \frac{d x}{n+x+N-2} \\
& =\log \left(\frac{n+i_{0}+N-2}{n+i+N-1}\right)^{1-(N-2) \alpha}
\end{aligned}
$$

Therefore

$$
\prod_{j=i_{0}}^{i}\left(1-\frac{2-2(N-2) \alpha}{n+2 j+N-2}\right)<\left(\frac{n+i_{0}+N-2}{i}\right)^{1-(N-2) \alpha}
$$

and

$$
\left|c_{n, 2 i}\right|<\frac{c}{n+2 i}\left(\frac{1}{i}\right)^{1-(N-2) \alpha}<c\left(\frac{1}{i}\right)^{2-(N-2) \alpha} .
$$

We conclude that

$$
\left|f_{n} S_{m n}\right|<\sum_{i=0}^{\infty}\left|c_{n, 2 i}\right| r^{n+2 i}<c \sum_{i=i_{0}}^{\infty}\left(\frac{1}{i}\right)^{2-(N-2) \alpha} .
$$

This is finite for $\alpha<1 /(N-2)$, and the proof Theorem 1 is complete.

2. To discuss the existence of $H D$-functions, we first prove:

LEMMA. For $\alpha \leq 0, f_{1}^{\prime}$ is bounded; for $\alpha>0, f_{1}^{\prime}(r)<$ const $\left(1-r^{2}\right)^{-(N-2) \alpha}$.

Proof. If $\alpha<0$, it is immediate from the estimates in the proof of Theorem 1 that $f_{1}^{\prime}$ is bounded. If $\alpha=0, d s=|d x|$ (the Euclidean metric), and $f_{1}^{\prime}=1<\infty$. If $\alpha>0, c_{1,2 i}>0$ for all $i$, and

$$
\begin{aligned}
c_{1,2 i}= & \prod_{j=1}^{i} \frac{(2 j-1)[2(N-2) \alpha+N-3+2 j]-(N-1)}{(2 j+1)(2 j+N-1)-(N-1)} \\
& <\prod_{j=1}^{i} \frac{(2 j-1)[2(N-2) \alpha+N-3+2 j]}{(2 j+N) 2 j} .
\end{aligned}
$$

For $N$ even,

$$
\begin{aligned}
c_{1,2 i} & <\prod_{j=1}^{i} \frac{2 j}{2 j+N} \cdot \prod_{j=1}^{i}\left(\frac{2 j-1}{2 j} \cdot \frac{2(N-2) \alpha+N-2+2 j-1}{2 j}\right) \\
& <\frac{2 \cdot 4 \cdots N}{(2 i+2)(2 i+4) \cdots(2 i+N)} \\
& \times \prod_{j=1}^{i}\left(\frac{2 j-1}{2 j} \cdot \frac{2(N-2) \alpha+N-2+2 j-2}{2 j-1}\right)
\end{aligned}
$$




$$
=\frac{2 \cdot 4 \cdots N}{(2 i+2)(2 i+4) \cdots(2 i+N)} \prod_{j=1}^{i} \frac{(N-2) \alpha+\frac{1}{2}(N-2)+j-1}{j} .
$$

Hence

$$
\begin{aligned}
& f_{1}^{\prime}(r)=1+\sum_{i=1}^{\infty}(2 i+1) c_{1,2 i} r^{2 i}<N+\sum_{i=1}^{\infty}(2 i+N) c_{1,2 i} r^{2 i} \\
&<N\left[1+2 \cdot 4 \cdots(N-2) \sum_{i=1}^{\infty}\left(\frac{1}{(2 i+2)(2 i+4) \cdots(2 i+N-2)}\right.\right. \\
&\left.\left.\quad \times \prod_{j=1}^{i} \frac{(N-2) \alpha+\frac{1}{2}(N-2)+j-1}{j}\right) r^{2 i}\right] .
\end{aligned}
$$

On the other hand,

$$
\left(1-r^{2}\right)^{-\left[(N-2) \alpha+\frac{1}{2}(N-2)\right]}=1+\sum_{i=1}^{\infty}\left(\prod_{j=1}^{i} \frac{(N-2) \alpha+\frac{1}{2}(N-2)+j-1}{j}\right) r^{2 i} .
$$

Repeating $\frac{1}{2}(N-2)$ times the process of first multiplying through by $r$ and then integrating, we obtain

$$
\begin{aligned}
& \text { const } \begin{aligned}
\left(1-r^{2}\right)^{-(N-2) \alpha}+\text { polynomial } \\
=\frac{r^{N-2}}{2 \cdot 4 \cdots(N-2)}[1+2 \cdot 4 \cdots(N-2) \\
\times \sum_{i=1}^{\infty}\left(\frac{1}{(2 i+2)(2 i+4) \cdots(2 i+N-2)}\right. \\
\left.\left.\quad \times \prod_{j=1}^{i} \frac{(N-2) \alpha+\frac{1}{2}(N-2)+j-1}{j}\right) r^{2 i}\right],
\end{aligned}
\end{aligned}
$$

which entails $f_{1}^{\prime}(r)<$ const $\left(1-r^{2}\right)^{-(N-2) \alpha}$.

For $N$ odd, the proof is the same except that now

$$
c_{1,2 i}<\frac{1 \cdot 3 \cdots N}{(2 i+1)(2 i+3) \cdots(2 i+N)} \prod_{j=1}^{i} \frac{(N-2) \alpha+\frac{1}{2}(N-1)+j-1}{j} .
$$

THEOREM 2. $B_{\alpha}^{N} \notin O_{H D} \Leftrightarrow|\alpha|<1 /(N-2), N \geq 3$.

Proof. We have

$$
D\left(f_{1} S_{1 m}\right)=\int_{0}^{1}\left(c_{1} f_{1}^{\prime}(r)^{2}+c_{2} r^{-2} f_{1}(r)^{2}\right)\left(1-r^{2}\right)^{(N-2) \alpha} r^{N-1} d r
$$

where $c_{1}$ and $c_{2}$ are positive constants. Since $f_{1}$ and $f_{1}^{\prime}$ are bounded for $\alpha \leq 0$,

$$
D\left(f_{1} S_{1 m}\right)<c \int_{0}^{1}\left(1-r^{2}\right)^{(N-2) \alpha} r^{N-3} d r<\infty
$$


for $-1 /(N-2)<\alpha \leq 0$. By the lemma,

$$
D\left(f_{1} S_{1 m}\right)<\int_{0}^{1}\left[d_{1}\left(1-r^{2}\right)^{-2(N-2) \alpha}+d_{2} r^{-2}\right]\left(1-r^{2}\right)^{(N-2) \alpha} r^{N-1} d r<\infty
$$

for $0<\alpha<1 /(N-2)$. Hence, $B_{\alpha}^{N} \notin O_{H D}$ if $|\alpha|<1 /(N-2)$. In view of $B_{\alpha}^{N} \in O_{G} \subset O_{H D}$ for $\alpha \geq 1 /(N-2)$, it remains to consider $\alpha \leq-1 /(N-2)$. Let $h$ be a nonconstant harmonic function. By means of the eigenfunction expansion of the restriction of $h$ to $|x|=r<1$, we obtain

$$
h=\sum_{n=0}^{\infty} \sum_{m=1}^{m_{n}} f_{n} a_{n m} S_{n m}
$$

on all of $B_{\alpha}^{N}$ with absolute and uniform convergence on compact subsets and with $a_{n m} \neq 0$ for some $(n, m), n>0$. By the Dirichlet orthogonality of spherical harmonics,

$$
\begin{aligned}
D(h) \geq a_{n m}^{2} D\left(f_{n} S_{n m}\right) & >a_{n m}^{2} \int_{B_{\alpha}^{N}}\left(1-r^{2}\right)^{-2 \alpha}\left(\frac{\partial f_{n} S_{n m}}{\partial r}\right)^{2} d V \\
& >c \int_{0}^{1} f_{n}^{\prime}(r)^{2}\left(1-r^{2}\right)^{(N-2) \alpha} d r .
\end{aligned}
$$

Here $f_{n}^{\prime}(r)$ converges as $r \rightarrow 1$, since the $c_{n, 2 i}$ are of constant sign for sufficiently large $i$. By virtue of $f_{n} S_{n m} \neq$ const, we have $f_{n}^{\prime}(r) \neq 0$ for $r>0$, and $\lim _{r \rightarrow 1} f_{n}^{\prime}(r) \neq 0$. Consequently

$$
D(h)>c \int_{\frac{1}{2}}^{1}\left(1-r^{2}\right)^{(N-2) \alpha} d r,
$$

which implies $B_{\alpha}^{N} \in O_{H D}$ for $\alpha \leq-1 /(N-2)$.

3. Let $O_{H B}^{N}, O_{H D}^{N}$ be the classes of Riemannian $N$-manifolds in $O_{H B}$, $O_{H D}$, respectively.

THEOREM 3. $O_{H B}^{N}<O_{H D}^{N}$ for every $N$.

Proof. The strict inclusion $O_{H B}^{2}<O_{H D}^{2}$ for Riemannian 2-manifolds is a trivial consequence of that for Riemann surfaces. In fact, endowing a Riemann surface by a conformal metric turns it into a Riemannian 2-manifold without affecting the harmonicity or the Dirichlet integral of a function on it.

For $N \geq 3$, the theorem follows from Theorems 1 and 2 . 


\section{REFERENCES}

[1] L. Ahlfors, Remarks on the classification of open Riemann surfaces, Ann. Acad. Sci. Fenn. Ser. A. I. No. 87 (1951), 8 pp.

[2] L. Ahlfors and H. L. Royden, A counterexample in the classification of open Riemann surfaces, Ann. Acad. Sci. Fenn. Ser. A. I. No. 120 (1952), 5 pp.

[ 3 ] D. Hada, L. Sario, and C. Wang, Dirichlet finite biharmonic functions on the Poincarè N-ball, J. Reine Angew. Math. (to appear).

[4] H. L. Royden, Some counterexamples in the classification of open Riemann surfaces, Proc. Amer. Math. Soc. 4 (1953), 363-370.

[5] L. Sario, Positive harmonic functions, Ann Arbor Conference on Analytic Functions (1953), Lectures on Functions of a Complex Variable, Univ. Mich. Press, Ann Arbor, 1955, 257-263.

[6] L. Sario and M. Nakai, Classification Theory of Riemann Surfaces, Springer, 1970, $446 \mathrm{pp}$.

[ 7 ] L. Sario, M. Schiffer, and M. Glasner, The span and principal functions in Riemannian spaces, J. Anal. Math. 15 (1965), 115-134.

[ 8 ] Y. Tôki, On the classification of open Riemann surfaces, Osaka Math. J. 4 (1952), 191-201.

[9] - On examples in the classification of Riemann surfaces, I. ibid. 5 (1953), 267-280.

University of California, Los Angeles 